\title{
sciendo
}

\section{Muscle Activity Asymmetry of the Lower Limbs During Sprinting in Elite Soccer Players}

\author{
by \\ Przemysław Pietraszewski ${ }^{1}$, Artur Gołaś ${ }^{1}$, Aleksander Matusiński ${ }^{1}$, \\ Sylwia Mrzygłód ${ }^{1}$, Aleksandra Mostowik ${ }^{1}$, Adam Maszczyk ${ }^{1}$
}

\begin{abstract}
The analysis of movement patterns through EMG activity provides the opportunity to identify the muscle groups most involved in a particular exercise, and to determine the scope of inter-limb deficiencies. The aim of the present study was to investigate the effects of a side-to-side muscle activity asymmetry between the left and the right lower limb during sprinting in soccer players. Sixteen professional soccer players took part in the study. Their age, body mass and body height equaled $23.7 \pm 7.6$ years, $81.2 \pm 10.8 \mathrm{~kg}$ and $179.3 \pm 12.2 \mathrm{~cm}$, respectively. The sprint test consisted of two maximal sprints over $30 \mathrm{~m}$ with a 5-min rest interval between each sprint. EMG was recorded bilaterally from the quadriceps, hamstrings and gluteal muscles. Regression analysis revealed a significant effect of a side-to-side average muscle activity asymmetry between the left and right hamstring (LH/RH) muscles during the speed tests at $5 \mathrm{~m}(p=0.044)$, and $30 \mathrm{~m}(p=0.045)$, as well as the left and right glutes $(L G / R G)$ at $5 \mathrm{~m}(p=0.044)$ and $30 \mathrm{~m}(p=0.043)$. Our results indicate that hamstring and glute muscles should be selectively and additionally activated during resistance training in soccer players to prevent injuries and improve sprint performance.
\end{abstract}

Key words: soccer, EMG, sprinting, movement pattern.

\section{Introduction}

The game of soccer is constantly evolving. In terms of physical requirements, the game has become significantly faster. For example, data from the English Premier League indicate that players at all positions cover greater distances at higher speeds than ever before. Often, a player performs 50 or more sprints in a match, and these actions can play a vital role in determining the outcome of the game (Haugen et al., 2014). Players are also required to change direction more than a thousand times per game, or approximately every 6-7 s. Typical high-speed movements performed in soccer are short in duration (1 to $5 \mathrm{~s}$ ). Considering the relatively short distance of soccer sprints, it seems logical that players develop acceleration capacity rather than maximum speed. For modern players, speed is the key component that contributes to the ultimate quality of performance (Small et al., 2010). A high number of intense high-speed actions such as accelerating, decelerating, jumping, turning and kicking (Bangsbo et al., 2006) require the hamstrings to perform in a position of extreme stretch. This means that significant muscle strength imbalances may increase muscular discomfort and result in a serious risk of injury.

One of the key elements of monitoring the training process includes the analysis of fundamental movement patterns, which serves as a basis for further development of athletes. The enhanced motor activation is a result of an increased number of active motor units and / or an increase in the frequency of their functional potential (Stastny et al., 2016). When describing motor activities the following terms are used: intramuscular coordination, i.e. the mutual relationship between the process of stimulation and inhibition of a single muscle, and intermuscular coordination, i.e. the participation

1 - Institute of Sport Sciences, The Jerzy Kukuczka Academy of Physical Education, Katowice, Poland. 
of all muscles in a given movement. Specific skills can affect the relationship between the strength of individual muscle groups (Crewther et al., 2005).

Electromyography (EMG) is a technique focused on the development, recording and analysis of myoelectric signals. Myoelectric signals are formed by physiological variations in the state of muscle fiber membranes. Kinesiological EMG can be described as the study of voluntary neuromuscular activation of muscles within postural tasks, functional movements, work conditions and treatment/training regimes (Van den Tillar and Ettema, 2013). Data collected from EMG analysis provides information on muscles' activity (Stastny et al., 2017). EMG data address to what extent a particular muscle is active in a certain exercise or task (Stastny et al., 2016). Another advantage of EMG is the ability to compare a side-to-side muscle activity, which provides the opportunity to identify the balance of muscle groups most involved in a motor activity (Enoka and Duchateau, 2015; Enoka and Fuglevand, 2001).

The development of textile electrodes embedded in clothing, which does not require the careful placement of sensors or wires that restrict movement (Scilingo et al., 2005), could provide a solution to the aforementioned limitations of traditional surface electrodes. Textile electrodes record EMG data from a larger area of the muscle and thus, excitation of whole muscle groups rather than individual muscles can be obtained. The use of EMG textile shorts allows to maintain game specific technique, enabling the analysis of the internal structure of movement under competitive conditions.

Soccer players may display unequal development of the right and left limbs due to certain technical actions performed in the sport, and such unequal development can cause functional or even structural asymmetries. A sideto-side asymmetry greater than $15 \%$ (Croisier et al., 2002; Croiser et al., 2008; Weber et al., 2010) may increase the risk of knee injuries. Movement patterns result from habits, typical activities, leg dominance and previous injuries (Cook, 2003; Coombs and Garbutt, 2002). Asymmetries between the muscles of the right and left leg below $9 \%$ indicate that these muscle groups are well balanced. When the difference in muscle activity between the right and left limb or side of the body falls between 9 and 18\% we can observe a trend towards asymmetry. Asymmetry greater than $18 \%$ indicates serious imbalances, and its origin should be defined and actions sought in order to avoid injuries (Alentorn-Geli, 2009; Colyer and McGuigan, 2018). The strength imbalance between the hamstring and quadriceps muscles is an essential predictor for hamstring strain (Croisier et al., 2002) and anterior cruciate ligament injuries (More et al., 1993).

The aim of the present study was to investigate the effects of a side-to-side muscle activity asymmetry between the lower limbs during sprinting of elite soccer players. It was hypothesized that the evaluation of muscle activity patterns during sprinting would allow to determine the internal structure of the movement under competitive conditions.

\section{Methods}

\section{Participants}

Sixteen elite soccer players took part in the study. Their age, body mass and body height equaled $23.7 \pm 7.6$ years, $81.2 \pm 10.8 \mathrm{~kg}$ and $179.3 \pm$ $12.2 \mathrm{~cm}$, respectively. Participants did not perform any strenuous exercises 48 hours prior to testing to avoid fatigue. Participants were informed verbally and in writing about the procedures, possible risks and benefits of the tests, and provided written consent before the commencement of the study. The study received the approval of the Bioethics Committee at the Academy of Physical Education in Katowice, Poland.

\section{Procedures}

The measurements were performed on a synthetic pitch during summer to minimize the effects of surface and weather conditions on performance. The sprint tests $(30 \mathrm{~m})$ were performed between 10 and $12 \mathrm{am}$. The test started with a standard 15 min warm-up, which included continuous running, joint mobility, static and dynamic stretching of the lower and upper limbs and 3-4 repetitions of $30 \mathrm{~m}$ runs at progressive speed until submaximal velocity was reached. The sprint test consisted of two maximal efforts of 30 $\mathrm{m}$ with a 5-min rest interval between each sprint. The sprint times at $5 \mathrm{~m}$ and $30 \mathrm{~m}$ were recorded using photocell gates (Witty, Microgate, Bolzano, Italy). Participants started the sprint $50 \mathrm{~cm}$ behind the first photocell gate. The fastest times at both 
distances were recorded for further analysis.

\section{Electromyography}

EMG was measured bilaterally from the quadriceps, hamstrings and gluteal muscles with shorts (Colyer and McGuigan, 2018) made of knitted fabric similar to elastic clothes used for sport activities or functional underwear, with the exception of EMG recordings from the skin surface (Myontec Ltd, Kuopio and Suunto Ltd, Vantaa, Finland). To measure the average rectified EMG signal, bipolar electrode pairs were located on the distal part of the quadriceps, hamstrings and glutes and the reference electrodes were placed longitudinally along the left and right lateral sides (over tractus iliotibialis) providing valid and repeatable data (Finni et al., 2007; Pesola et al., 2014; Tikkanen et al., 2014). The electrodes located on quadriceps muscles collected data from $\mathrm{m}$. vastus lateralis, $\mathrm{m}$. vastus medialis and $\mathrm{m}$. rectus femoris. The vastus intermedius muscle is located deeply under the other muscles and thus was not included. Electrodes located on the hamstring muscles recorded the signal from the biceps femoris, semimembranosus and semitendinosus. Electrodes placed on the gluteal muscles recorded signals from the gluteus maximus and gluteus medius muscles. EMG signals were recorded in a raw form with a sampling frequency of $1000 \mathrm{~Hz}$ and a frequency band of $50 \mathrm{~Hz}-200 \mathrm{~Hz}(-3 \mathrm{~dB})$. The signal gain factor prior to $\mathrm{A} / \mathrm{D}$ conversion was 1220 and for the A/D-conversion a 12-bit. The raw EMG signals were first rectified and then averaged over each $100 \mathrm{~ms}$ intervals without overlapping. Thus 10 consecutive samples from raw signals created one rectified, averaged data sample. These $10 \mathrm{~Hz}$ data were stored in the ASCII format in the memory of the module, which was downloaded to a PC using custom software. From the $10 \mathrm{~Hz}$ EMG data ARV was calculated from a $1 \mathrm{~s}$ window during a stable torque signal (Finni et al., 2007). Six channels (three from each leg) were recorded. EMG signals were transferred to the data collection unit by MCell in a high precision manner. By default, each signal channel was drawn 25 times a second $(25 \mathrm{~Hz})$. To ensure proper signal conduction, the electrodes were moisturized with tap water before dressing on the shorts. When wetted, a membrane covering the electrodes prevented the electrodeskin interface from drying. Before the completion of all the tests in a single day, 2-3 s evaluations of the Maximal Voluntary Contraction (MVC) of the sprint prime movers were performed according to the SENIAM procedure (Tillar and Ettema, 2013). These evaluations were performed in order to normalize electromyographic records.

\section{Statistical analysis}

The data were processed using Statistica software and presented as means with standard deviations. Shapiro-Wilk, Levene and Mauchly's tests were used to verify the normality and homogeneity of the sample's data variances, respectively. To find the most important predictors for the dependent variable a regression analysis was used (Maszczyk et al., 2014, 2016). Statistical significance was set at $p<0.05$.

\section{Results}

A side-to-side average muscle activity asymmetry between the left and right quadriceps (LQ and $\mathrm{RQ}$ ), hamstrings ( $\mathrm{LH}$ and $\mathrm{RH}$ ) and glutes (LH and $\mathrm{RH}$ ) is presented in Table 1.

A side-to-side asymmetry was lowest between the left and right quadriceps (7.9\%), while the largest asymmetry was visible between the right and left glutes (17.5\%). The Hamstring/Quadriceps Ratios recorded during the sprint test $(5 \mathrm{~m}$ and $30 \mathrm{~m})$ are presented in Table 2.

Regression analysis revealed a significant effect of a side-to-side average muscle activity asymmetry between left and right hamstrings $(\mathrm{LH} / \mathrm{RH})$ muscles during speed tests at $5 \mathrm{~m}(p=$ $0.044)$, and $30 \mathrm{~m}(p=0.045)$ and left and right glutes $(\mathrm{LG} / \mathrm{RG})$ at $5 \mathrm{~m}(p=0.044)$ and $30 \mathrm{~m}(p=$ $0.043)$.

\section{Discussion}

The objective of this study was to compare the side-to-side muscle activity asymmetry between the lower limbs during sprinting of elite soccer players. We detected the greatest side-to-side average muscle activity asymmetry between the left and right glutes muscles (17.5\%), and the left and right hamstrings (9.8\%). Therefore, it seems reasonable to assume that performing unilateral strength training exercises may be important to increase the efficiency of bilateral movements, promoting contralateral effects and decreasing the risk of injury (Carroll et al., 2006; Fousekis et al., 2010). As mentioned, strength and conditioning coaches 
should promote unilateral training (e.g. split squats, forward and reverse lunges, single-leg dead lifts, single leg back extensions, etc.) to correct bilateral asymmetry in professional soccer players with substantial bilateral differences. The dominance of one side of the body over the other causes differences in strength of various muscles activated during specific actions required in the sport. Some studies of soccer players have shown differences in strength and flexibility between the dominant and the non-dominant leg (Carroll et al., 2016; Daneshjoo et al., 2013). However, all of these studies are based on the analysis of the external structure of the movement (isokinetic assessment of velocity and acceleration) and not through actual muscular activity under competitive conditions (EMG during specific movements). However, differences of movement patterns between isokinetic assessments and sport specific tasks may lead to conflicting results in the identification of bilateral asymmetries. When interpreting thresholds of the asymmetry, a limb difference $\geq 15 \%$ has been shown to negatively impact function and performance following injury. Additionally, an increased side-to-side asymmetry between preferred and non-preferred limbs may also increase potential risks for knee injuries (Kong and Burns, 2010; Tol et al., 2014).

\section{Table 1}

A side-to-side average muscle activity asymmetry between left and right quadriceps ( $L Q / R Q)$, hamstrings $(L H / R H)$, and glutes $(L G / R G)$.

The mark - before the value indicates greater activity on the right side.

\begin{tabular}{cccc}
\hline & LQ/RQ (\%) & LH/RH (\%) & LG/RG (\%) \\
\hline 1 & -0.6 & -13.4 & -14.0 \\
2 & 5.4 & 14.6 & 12.0 \\
3 & 1.8 & 7.2 & 4.8 \\
4 & -3.6 & 7.6 & 27.8 \\
5 & -10.6 & 15.2 & -8.8 \\
6 & 4.8 & 14.6 & 12.0 \\
7 & -2.6 & -30.6 & -20.8 \\
8 & 20.0 & 19.0 & 21.0 \\
9 & -18.0 & 3.9 & -19.8 \\
10 & 13.8 & 4.2 & 19.6 \\
11 & 15.4 & 4.8 & 20.4 \\
12 & -3.8 & -7.8 & 2.0 \\
13 & -0.8 & -3.4 & 42.6 \\
14 & -13.4 & -6.6 & -27.4 \\
15 & 5.4 & 3.4 & 6.4 \\
16 & -6.0 & -0.2 & 20.0 \\
\hline SD & $7.9 \pm 6.4$ & $9.8 \pm 7.7$ & $17.5 \pm 10.2$ \\
\hline
\end{tabular}


Table 2

Results of the $5 \mathrm{~m}$ and $30 \mathrm{~m}$ sprint tests.

\begin{tabular}{ccc}
\hline & $5 \mathrm{~m}(\mathrm{~s})$ & $30 \mathrm{~m}(\mathrm{~s})$ \\
\hline 1 & 1.09 & 4.10 \\
2 & 1.04 & 4.11 \\
3 & 1.05 & 4.10 \\
4 & 1.05 & 3.99 \\
5 & 1.03 & 4.00 \\
6 & 1.11 & 4.28 \\
7 & 1.07 & 4.18 \\
8 & 1.10 & 4.15 \\
9 & 1.12 & 4.26 \\
10 & 1.11 & 4.18 \\
11 & 1.17 & 4.31 \\
12 & 1.06 & 4.22 \\
13 & 1.05 & 4.08 \\
14 & 1.15 & 4.13 \\
15 & 0.97 & 3.98 \\
16 & 0.93 & 3.97 \\
\hline SD & $1.1 \pm 0.1$ & $4.1 \pm 0.1$ \\
\hline
\end{tabular}

Regression analysis revealed a significant effect of a side-to-side average muscle activity asymmetry between left and right hamstrings $(\mathrm{LH} / \mathrm{RH})$ and glutes $(\mathrm{LG} / \mathrm{RG})$ during sprinting at 5 and $30 \mathrm{~m}$. Many coaching authorities indicate that hamstrings and glute muscles play a significant role in sprinting, yet this has not been confirmed by empirical studies which compare the muscle activity asymmetry between the limbs during running at high speeds (Cook, 2010; Jeffreys, 2008). Most soccer players have a preferred leg for kicking the ball, and it is believed that this preference may lead to an asymmetry in strength and flexibility of the lower extremities. Daneshjoo et al. (2013) attempted to determine whether strength and flexibility asymmetries were present in the legs of soccer players. They found significant differences between the preferred and non-preferred leg in the knee flexors and for the dynamic control ratio. In both cases the knee flexors of the preferred leg were weaker than those of the non-preferred leg. Almost $68 \%$ of players had significant musculoskeletal abnormalities (imbalance $>10 \%$ ) in one or more muscle groups, while no significant differences were found in flexibility of the hip joint between the preferred and non-preferred leg. Our results indicate that the greatest imbalance between the right and left side of the body occurs in the glute muscles and the smallest in the quadriceps 
muscles. These results show a greater need for activating the glute and hamstring muscles during resistance training of soccer players. The average activity in hamstring muscles is 33\% greater in comparison to the quadriceps muscles. A much greater activity of hamstring muscles compared to the quadriceps during sprinting confirms their greater involvement in this motor task. A limitation of this study relates to the lack of evaluation of the external movement structure. For future research we suggest to determine the relationship between the internal and external structure of movement during sprinting and other specific activities in soccer. Another limitation of the study is the lack of muscle activity analysis of the gastrocnemius and tibialis muscles, which seems to play a significant role in sprinting.

\section{Conclusions}

The novel aspect of this research included the analysis of EMG activity of the prime movers of the lower limbs during sprinting of elite athletes in both limbs to detect functional asymmetries. Most EMG studies on athletes are performed in accordance with Seniam recommendations on one side of the body (dominant side), what may by a significant limitation.

For practical reasons we suggest evaluating the $\mathrm{H} / \mathrm{Q}$ ratio through the inner structure of movement during natural activities, specific for particular team sport games. Our results indicate that the greatest imbalance between the right and left lower limbs occurs in the glute muscles and the smallest in the quadriceps muscles. This implies that hamstring and glute muscles should be selectively and additionally activated during resistance training in soccer players to prevent injuries and improve sprint performance.

\section{Acknowledgements}

This study was made possible by the financial support of the Jerzy Kukuczka Academy of Physical Education in Katowice.

\section{References}

Alentorn-Geli E, Myer GD, Silvers HJ, Samitier G, Romero D, Lázaro-Haro C, Cugat R. Prevention of noncontact anterior cruciate ligament injuries in soccer players. Part 1: Mechanisms of injury and underlying risk factors. Knee Surg Sports Traumatol Arthrosc, 2009; 17(7): 705-29

Bangsbo J, Mohr M, Krustrup P. Physical and metabolic demands of training and match-play in the elite football player. J Sports Sci, 2006; 24(7): 665-674

Colyer SL, McGuigan PM. Textile Electrodes Embedded in Clothing: A Practical Alternative to Traditional Surface Electromyography when Assessing Muscle Excitation during Functional Movements. J Sports Sci Med, 2018; 17(1): 101-109

Cook G. Movement: Functional movement systems: Screening assessment and corrective strategies. 2010, Aptos, CA: On Target

Coombs R, Garbutt G. Developments in the use of the hamstring/quadriceps ratio for the assessment of muscle balance. J Sports Sci Med, 2002; 1(3): 56-62

Crewther B, Cronin J, Keogh J. Possible stimuli for strength and power adaptation - Acute mechanical responses. Sports Med, 2005; 35: 967-989

Croisier J, Forthomme B, Namurois MH, Vanderthommen M, Crielaard JM. Hamstring muscle strain recurrence and strength performance disorders. Am J Sports Med, 2002; 30(2): 199-203

Croisier J, Ganteaume S, Binet J, Genty M, Ferret JM. Strength imbalances and prevention of hamstring injury in professional soccer players: a prospective study. Am J Sports Med, 2008; 36(8): 1469-75

Daneshjoo A, Rahnama N, Mokhtar AH, Yusof A. Bilateral and Unilateral Asymmetries of Isokinetic Strength and Flexibility in Male Young Professional Soccer Players. J Hum Kinet, 2013; 36: 45-53

Enoka RM, Duchateau J. Inappropriate interpretation of surface EMG signals and muscle fiber characteristics impedes understanding of the control of neuromuscular function. J Appl Physiol, 2015; 119: 1516-1518

Enoka RM, Fuglevand AJ. Motor unit physiology: some unresolved issues. Muscle Nerve, 2001; 24: 4-17

Finni T, Hu M, Kettunen P, Vilavuo T, Cheng S. Measurement of EMG activity with textile electrodes embedded into clothing. Physiol Meas, 2007; 28(11): 1405-19 
Fousekis K, Tsepis E, Vagenas G. Lower Limb Strength in Professional Soccer Players: Profile, Asymmetry, and Training Age. J Sports Sci Med, 2010; 9(3): 364-373

Haugen T, Tonnessen E, Hisdal J, Seiler S. The role and development of sprinting speed in soccer. Int J Sports Physiol Perform, 2014; 9(3): 432-41

Jeffreys, I. Movement training for field sports: Soccer. Strength Cond J, 2008; 30(4): 19-27

Kong PW, Burns SF. Bilateral difference in hamstrings to quadriceps ratio in healthy males and females. Phys Ther Sport, 2010; 11: 12-17

Maszczyk A, Gołaś A, Pietraszewski P, Roczniok R, Zając A, Stanula A. Application of Neural and Regression Models in Sports Results Prediction. Procedia - Soci Behavio Sci, 2014; 117: 482-487

Maszczyk A, Golas A, Czuba M, Krol H, Wilk M, Stastny P, Goodwin J, Kostrzewa M, Zajac A. EMG Analysis and Modelling of Flat Bench Press Using Artificial Neural Networks. Sajrper, 2016; 38(1): 91103

Small K, McNaughton L, Greig M, Lovell R. The effects of multidirectional soccer-specific fatigue on markers of hamstring injury risk. J Sci Med Sport, 2010; 13(1): 120-5

Stastny P, Tufano JJ, Golas A, Petr M. Strengthening the Gluteus Medius Using Various Bodyweight and Resistance Exercises. Strength Cond J, 2016; 38: 91-101

Tikkanen O, Kärkkäinen S, Haakana P, Kallinen M, Pullinen T and Finni T. EMG, heart rate, and accelerometer as estimators of energy expenditure in locomotion. Med Sci Sports Exerc, 2014; 46: 18311839

Tol JL, Hamilton B, Eirale C, Muxart P, Jacobsen P, Whiteley R. At return to play following hamstring injury the majority of professional football players have residual isokinetic deficits. Br J Sports Med, 2014; 48(18): 1364-1369

van den Tillaar R, Ettema G. A comparison of muscle activity in concentric and counter movement maximum bench press. J Hum Kinet, 2013; 38: 63-71

\section{Corresponding author:}

\section{Przemysław Pietraszewski PhD.}

Institute of Sport Sciences;

The Jerzy Kukuczka Academy of Physical Education, Katowice, Poland

Mikołowska 72, 40-065 Katowice, Poland

Phone: +48727444444

E-mail: p.pietraszewski@awf.katowice.pl 